\title{
ALIMENTAÇÃO E DISTRIBUIÇÃO ESPACIAL DE ALGUMAS ESPÉCIES DE PEIXES DO IGARAPÉ DO CANDIRÚ, AMAZONAS, BRASIL.
}

\author{
Cláudia. P. D. SILVA ${ }^{1}$
}

\begin{abstract}
RESUMO - O conteúdo estomacal de 29 espécies de peixes e a distribuição destas em diferentes microhabitats existentes em um igarapé de terra-firme, igarapé do Candirú, na Amazônia Central, foram analisados. Sete tipos de microhabitats foram ob_ervados e algumas espécies apresentaram estruturas morfológicas adaptativas que eram adequadas à captura do alimento consumido e ao microhabitat onde foram sempre coletadas. A relação entre a dieta alimentar e o microhabitat no qual a espécie permanecia, foi observada. Outras espécies apresentaram mudanças na escolha do microhabitat em relação ao seu estágio de desenvolvimento, embora não tenha sido observada alteração quanto ao tipo de alimento consumido. Neste caso é possível que a diminuição no risco de predação seja o fator principal influenciando na mudança de microhabitat. Neste trabalho são discutidos alguns prováveis fatores que influenciam as espécies na escolha de um determinado microhabitat a ser explorado.
\end{abstract}

Palavras-chave: Distribuição espacial, alimentação, peixes, igarapé, Amazônia.

Feeding and Spacial Distribution of Some Species of Fishes in Igarape Candiru, Amazonia, Brazil SUMMARY - Stomach contents of 29 species of fish and the distribution of these species in different microhabitats in a stream (igarapé do Candirú) in Central Amazonia, were analysed. Seven different types of microhabitats were observed and some species presented adaptatives morphological structures that combined with feeding habits and microhabitat in which the species was always colected. The existence of the relationship between feeding habits and microhabitat was observed. Other fish species changed microhabitat according to their state of development eventhough no change occurred in their feeding habits. In these cases, it is presumed that predation exercised greater control over distribution than did feeding. In the present work, the probable factors that influence fish species in their choice of microhabitat to be exploited are discussed.

Key words: Spacial distribution, feeding, fishes, stream, Amazonia.

\section{INTRODUÇÃO}

As comunidades de peixes de água doce tropicais apresentam padrões caracteristicos quanto à utilização dos recursos ambientais.

Peixes de rios, por exemplo, apresentam-se muito móveis, várias espécies realizam grandes migrações tróficas e/ou reprodutivas. Em outros sistemas aquáticos, como por exemplo pequenos córregos e igarapés, as comunidades de peixes são mais fixas, ou seja, a maioria das espécies que às compõem não realiza longas migrações, passando quase todo seu tempo de vida no mesmo sistema ou habitat. De modo geral essas espécies tendem a se especializar em um determinado espaço onde as condições ambientais

INPA - Alameda Cosme Ferreira, 1756 - Manaus, AM. 
apresentam-se mais uniformes e adequadas para elas (LOWEMCCONNELL, 1975; ZARET \& RAND, 1971; GORMAN \& KARR, 1978). Estes espaços físicos são denominados por GOODALL (1986) de biótopos ou microhabitats e são considerados como unidades de um habitat, onde os indivíduos de uma espécie são capazes de se estabelecerem e completarem seus ciclos de vida.

Os trabalhos realizados com comunidades de peixes em pequenos córregos tropicais (ZARET \& RAND, 1971; SAUL, 1975; SOARES, 1979; VIANNA, 1989; RINNE, 1992; SILVA, 1992) indicam que algumas espécies apresentam preferência por um tipo de microhabitat, ou seja. muitas espécies são típicas de determinados ambientes. São poucos, entretanto, os trabalhos que visam explicar quais os prováveis fatores que influenciam as espécies a ocuparem diferentes espaços. Para CONNELL (1975) as espécies se distribuem em habitats disponiveis dependendo de três fatores: disponibilidade do alimento, intensidade da predação e tolerância às condições físico-químicas ambientais. RINNE (1992) sugeriu que a história evolutiva das espécies ou as interações competitivas interespecificas poderiam explicar as diferenças na escolha de microhabitat entre sete espécies do rio Aravaipa, no Arizona. É possivel que os fatores relacionados com a escolha dos microhabitats variem de um local para outro, contudo é geral a observação de que algumas espécies são comuns em determinados habitats e microhabitats e que muitas delas apresentam adaptações morfológicas que promovem maior e melhor exploração de um determinado recurso.

Neste trabalho, analisamos a distribuição de 29 espécies de peixes em sete diferentes microhabitats e seus hábitos alimentares, tentando correlacioná-los. São discutidos alguns fatores que poderiam estar influenciando esta distribuição.

\section{ÀREA DE ESTUDO}

O estudo foi realizado no igarapé do Candirú (Fig. 1), pertencente à bacia do rio Puraquequara. $\mathrm{O}$ local de coletas situou-se a $2^{\circ} 45^{\prime} 25^{\prime \prime} \mathrm{S}$ e $59^{\circ} 52^{\prime}$ W. A área do igarapé está asssentada em sedimentos terciários da Amazônia Central, nos baixos platôs sedimentares denominados "Formação Barreira". Há predominância de Latossolo Amarelo Distrófico constituido dos minerais caolinita, quartzo, óxidos e hidróxidos de ferro e aluminio (CHAUVEL, 1981).

O clima é do tipo submetaxérico (1 a 20 dias biologicamente secos) (RADAMBRASIL, 1978). Em relação à cobertura vegetal, é do tipo floresta tropical densa.

O igarapé do Candirú possui aproximadamente $7 \mathrm{~km}$ de extensão com largura e profundidade médias de 9,0 e 1,5 metros, respectivamente. A vegetação marginal é tipicamente de floresta primária. A água é de cor clara, com condutividade média de 8 $\mu \mathrm{S} / \mathrm{cm}, \mathrm{pH}$ de 5,0 e temperatura de 


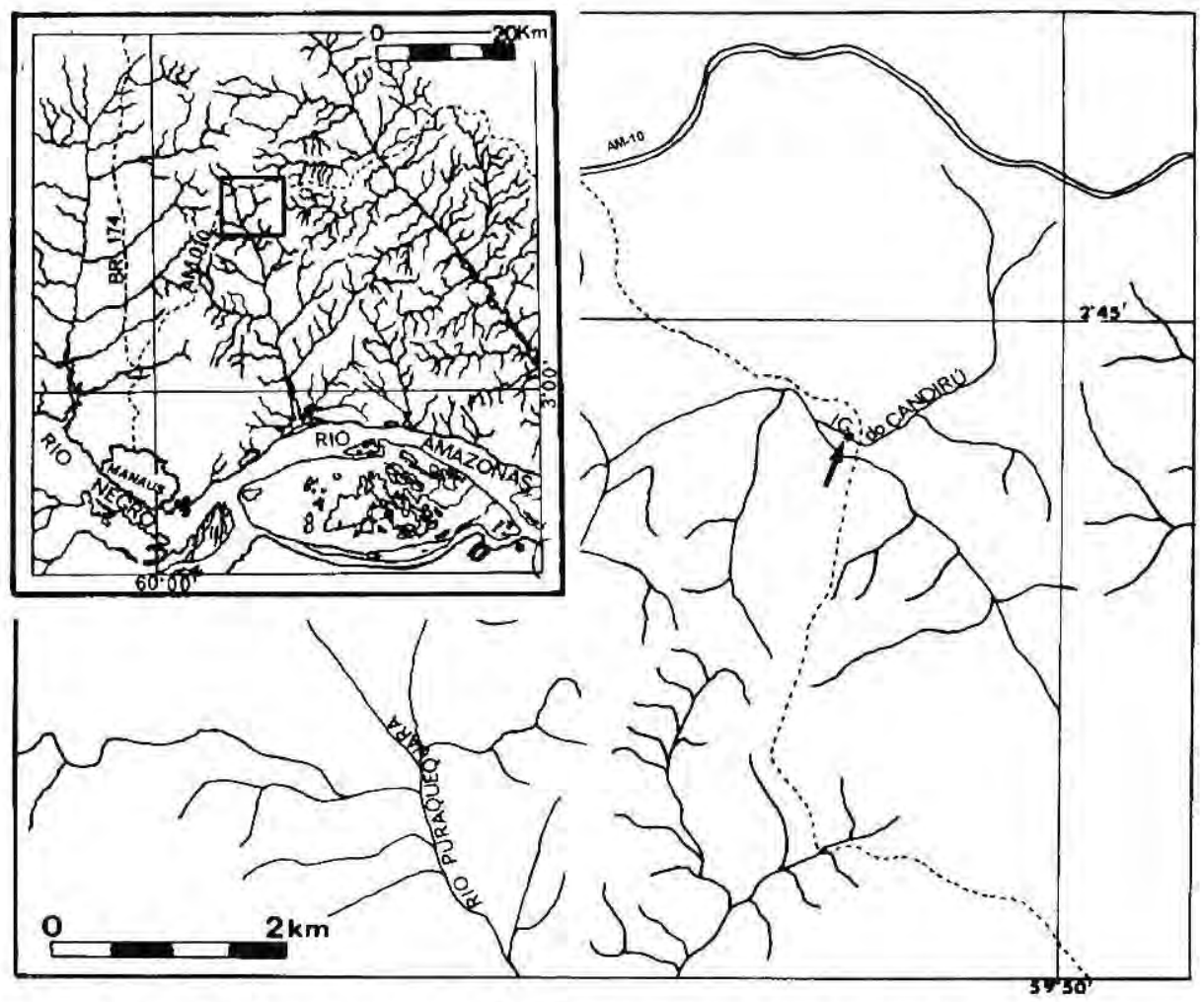

Figura 1. Localização da área de estudo. A seta indica o local de coleta.

$25^{\circ} \mathrm{C}$. Na área de coleta, o dossel apresenta-se mais aberto, conferindo boa luminosidade ao leito do igarapé, o que permite o desenvolvimento de algas filamentosas como Spirogyra sp., Ulotrix sp. (Chlorophytas) e Batrachospermum sp. (Rodophyta). Estas algas são encontradas sobre o leito, próximo às margens e/ou sobre folhas de macrófitas aquáticas, servindo de fonte de alimento para alguns peixes herbivoros. Cyperaceae semiaquática, enraizada no fundo, com folhas e inflorescências aéreas, faz-se presente nas margens do igarapé, além de Tonina sp. (Eriocaulaceae). As margens com vegetação permanecem invadidas pelas águas, proporcionando local para esconderijo, de peixes de pequeno porte e de alevinos. Nos meses de estiagem, há diminuição da área marginal alagada, porém estas não secam completamente.

Durante as coletas preliminares, observamos que certas espécies de peixes eram sempre coletadas em determinados biótopos. Deste modo, foi possível distinguir e caracterizar sete tipos de microhabitats (Tab. 1).

\section{MATERIAIS E MÉTODOS}

A periodicidade das coletas foi determinada baseando-se na pluviometria da região (RIBEIRO \& ADIS, 1984). Foram realizadas quatro 
Tabela 1. Características fisicas dos sete microhabitats observados no igarapé estudado.

\begin{tabular}{|c|c|c|c|c|}
\hline MICROHABITAT & $\begin{array}{l}\text { VELOCIDADE } \\
\text { DA ÁGUA }(\mathrm{m} / \mathrm{s})\end{array}$ & $\begin{array}{l}\text { PROFUNDIDADE } \\
(\mathrm{cm})\end{array}$ & $\begin{array}{c}\text { TIPO } \\
\text { DE SUBSTRATO }\end{array}$ & OBSERVAÇŌES \\
\hline Moita de Cyperaceae & 0,15 & 65 & Folhas de Cyperaceae & $\begin{array}{l}\text { Algas filamentosas sāo } \\
\text { constantemente encontra- } \\
\text { das sobre as folhas desta } \\
\text { macrófita }\end{array}$ \\
\hline Fundo arenoso & 0,05 & 30 & Areia & - \\
\hline Água livre & 0,10 & 96 & - & - \\
\hline Alagado marginal & 0 & 12 & sedimento fino (argila) & $\begin{array}{l}\text { Gramineas de cerca de } 20 \\
\text { cm de altura crescem neste } \\
\text { local. A pouca profundida- } \\
\text { de e baixa velocidade da } \\
\text { àgua, promove elevaçäo da } \\
\text { temperatura em cerca de } \\
3^{\circ} \mathrm{C} \text { a mais do que no leito } \\
\text { principal. }\end{array}$ \\
\hline Margem sombreada & 0 & 66 & folhiço & - \\
\hline Moita de Tonina sp. & 0,05 & 20 & - & $\begin{array}{l}\text { Esta macrófita se desenvol- } \\
\text { ve em áreas proximas ás } \\
\text { margens permanecendo } \\
\text { parte da planta submersa e } \\
\text { outra parte aérea. Entretan- } \\
\text { to alguns bancos foram } \\
\text { observados no leito do } \\
\text { igarapé, onde em algumas } \\
\text { épocas estavam completa- } \\
\text { mente submersas. }\end{array}$ \\
\hline Tronco submerso & 0,10 & 44 & & $\begin{array}{l}\text { Os peixes ficam escondi- } \\
\text { dos nas cavidades dos } \\
\text { troncos. }\end{array}$ \\
\hline
\end{tabular}

coletas no período de chuva (marçomaio-dezembro/90 e fevereiro/91) e três no período de estiagem (julhoagosto-outubro/90).

Os peixes foram coletados utilizando-se rede de cerco de três metros de comprimento por um metro de largura e,malha de três milimetros entre nós. Nas poças marginais utilizamos também o puçá. As coletas foram realizadas em um trecho do igarapé de cerca de $400 \mathrm{~m}^{2}$. As espécies foram separadas por local de captura e assumimos que elas estavam ocupando seus biótopos naturais, baseando-nos em observações de campo e em SAUL (1975), SOARFS (1979), HENDERSON \& W $/$ LKER (1990) e SANTOS (1990) que também capturaram algumas espécies em locais com caracteristicas semelhantes àquelas encontradas por nós. As observações locais e a frequência na captura de uma espécie em um tipo de biótopo, como suposição de seu lugar de vida, ocorre também em estudos de comunidades de pássaros (CODY, 1968). Desta forma, nos certificamos de estarmos coletando os peixes no seu local de vida.

Procuramos explorar ao máxımo todos os microhabitats amostrados, cercando-os com a rede enquanto se fazia batimentos na água, na área cercada, a fim de desalojar espécimens que ficavam escondidos.

Os peixes capturados foram fixados no campo em solução de fo mol a $10 \%$. No laboratório foram lavados em água corrente e posteriormente conser- 
vados em álcool a $70 \%$. Os peixes foram identificados segundo GÉRY (1977), KULLANDER (1986) e COSTA (1990 a, b) e o conteúdo estomacal foi analisado em microscópio estereoscópio, apenas para presença ou ausência dos ítens alimentares. A identificação dos itens alimentares baseou-se em CHU (1949) e MACAN (1959). As algas e alguns insetos aquáticos foram identificados por especialistas do INPA.

\section{RESULTADOS}

\section{Alimentação}

Neste estudo consideramos como ítens alóctones os Orthoptera e Hymenoptera (principalmente Formicidae) e como ítens autóctones os peixes, Coleoptera, Decapoda, Odonata, Diptera (principalmente Chironomidae), Collembola, Trichoptera, algas, microcrustáceos (principalmente Copepoda Harpacticoida), ácaros e detritos. $\mathrm{Na}$ Tabela 2 são apresentados os números de indivíduos de cada espécie que estavam se alimentando de cada item.

Das espécies que tiveram o conteúdo estomacal analisado, apenas Pyrrhulina laeta, Carnegiella strigata, Rivulus urophthalmus e Rivulus ornatus se alimentaram exclusivamente de ítens alóctones (Hymenoptera).

Todas essas espécies possuem a boca em posição superior, o que facilita a captura de organismos na superficie da água. Apistogramma sp., Ancistrus sp., Farlowella sp., Rineloricaria sp. e Hoplias malabaricus ingeriram apenas ítens autóctones, sendo que a maioria se alimentou de um só ítem, exceto Ancistrus sp. que de 15 exemplares observados, dois alimentaram-se de algas e de Diptera ao mesmo tempo. Os dois exemplares de Apistogramma $\mathrm{sp}$. coletados, apresentaram somente detrito nos estômagos. Farlowella sp. e Rineloricaria sp. apresentaram exclusivamente algas filamentosas nos estômagos. Doze espécies (Characidium pteroides, Ammocryptocharax sp., Nannostomus marginatus, Copella nattereri, Iguanodectes geisleri, Bryconops caudomaculatus, Bryconops melanurus. Hyphessobrycon sp., Hemigrammus sp1, Hemigrammus sp2, Hypselecara coryphaenoides e Aequidens pallidus) apresentaram maior variedade de itens alimentares, tanto de origem alóctone como autóctone.

Em algumas espécies, não foi possível determinar a origem dos ítens alimentares encontrados nos estômagos.

\section{Distribuição espacial}

$\mathrm{Na}$ Tabela 3 estão indicados os locais de captura das espécies.

Nos bancos de Cyperaceae aquática, foram capturadas seis espécies, dentre as quais Rineloricaria sp. e Farlowella sp. só apareceram neste biótopo. Suas características morfológicas tais como corpo comprido e estreito, boca inferior e em forma de ventosa, permitiram que as mesmas permanecessem sobre essas macrófitas e se alimentassem das algas que ficavam presas sobre suas folhas.

No fundo arenoso só foi capturado C. pteroides que também apresenta adaptações morfológicas. Esta espécie possui as nadadeiras peitorais bem 
Tabela 2. Ocorrência dos ítens alimentares nos estômagos das espécies estudadas. ORT=Orthoptera; HYM=Hymenoptera; PEI=Peixe; $\mathrm{COL}=$ Coleoptera; $\mathrm{DEC}=$ Decapoda; $\mathrm{ODO}=$ Odonata; $\mathrm{DIP}=$ Diptera; $\mathrm{CLB}=$ Collembola; $\mathrm{TRI}=$ Trichoptera; $\mathrm{ACA}=\mathrm{A}$ caro; $\mathrm{MIC}=\mathrm{Microcrustáceo,} \mathrm{ALG}=\mathrm{Alga}$; $\mathrm{RI}=$ Restos de insetos; $\mathrm{RV}=$ Restos de vegetais; $\mathrm{ONI}=$ Origem não identificada; $\mathrm{N}=$ Número de estômagos observados.

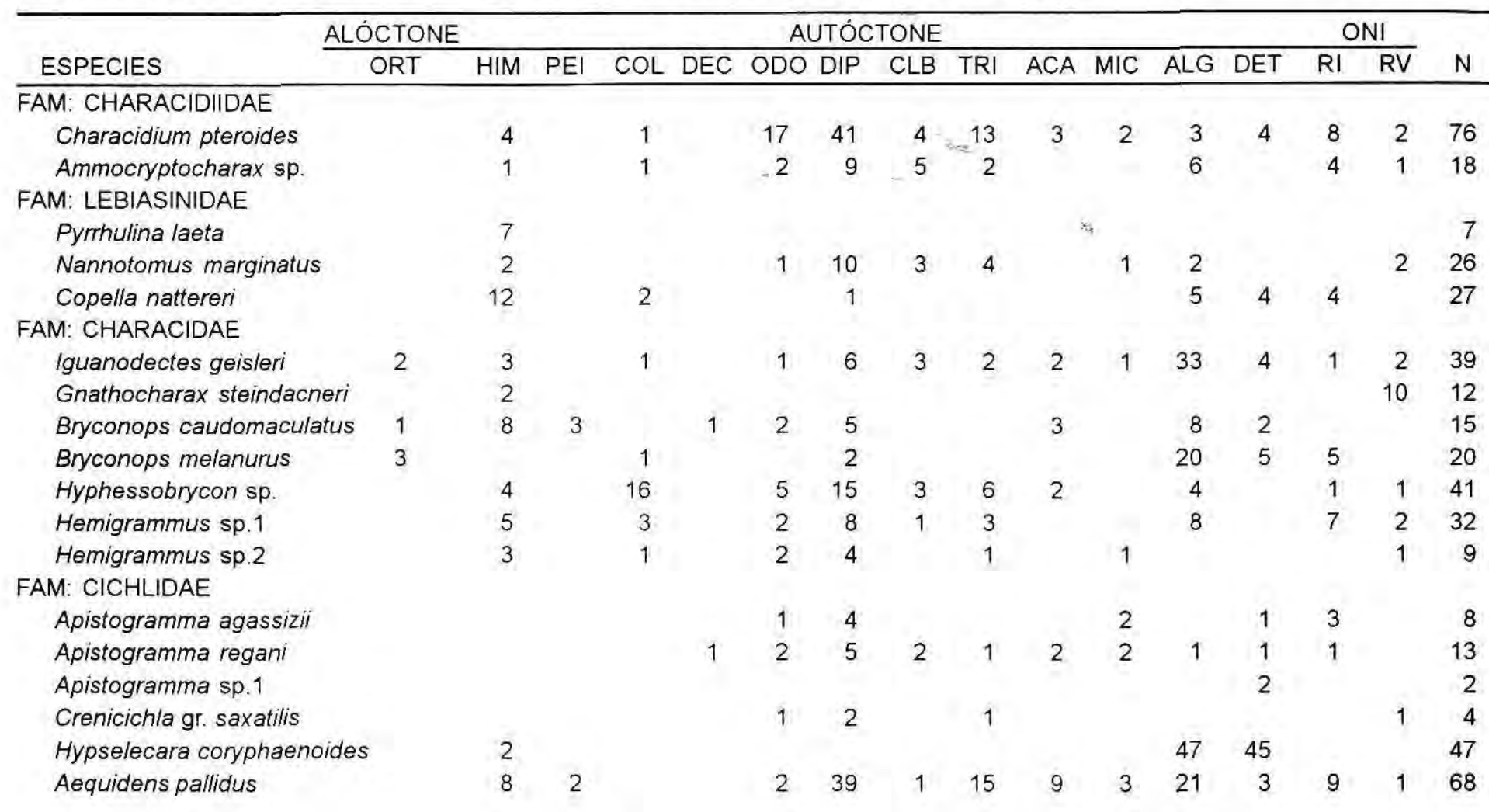


Cont.

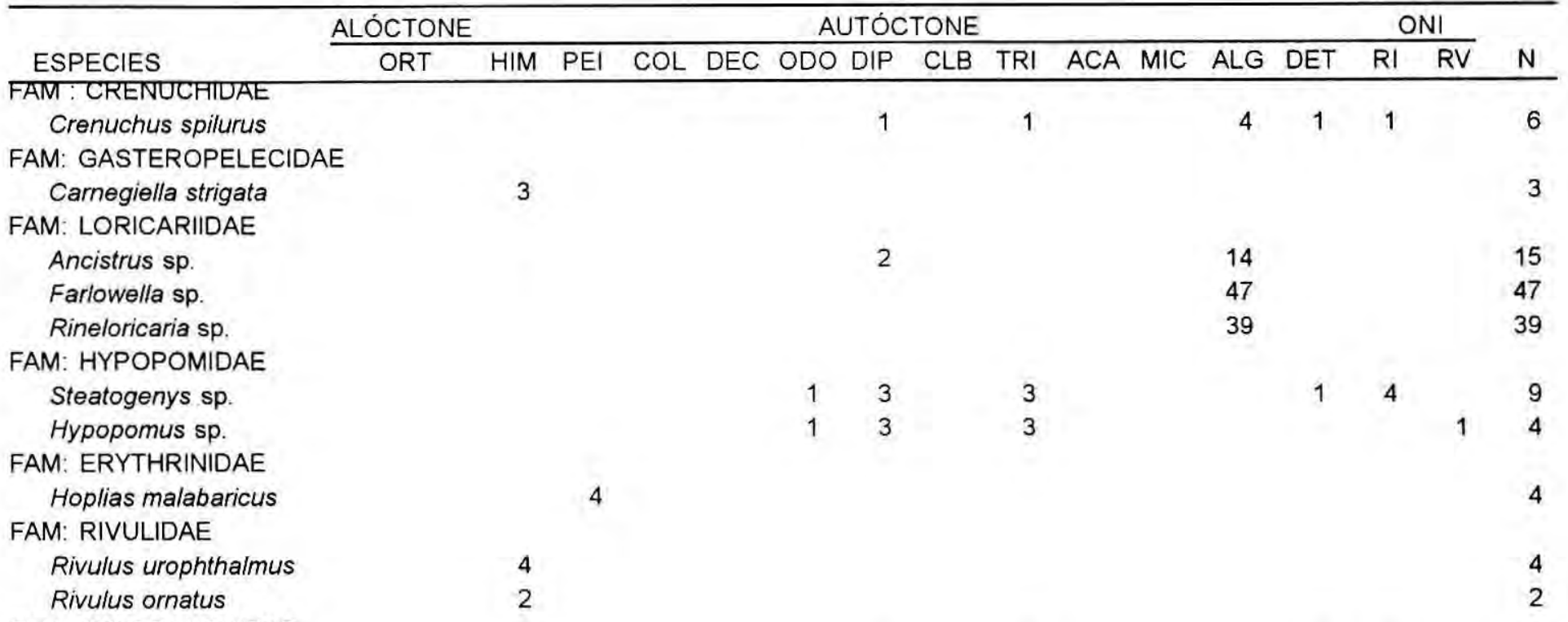

FAM; SYNBRANCHIDAE

Synbranchus marmoratus

11

10

67

15 
Tabela 3. Local de captura das espécies estudadas. $1=$ Moita de Cyperaceae; $2=$ Fundo arenoso; 3=Água livre; 4=Alagado marginal; 5=Margem sombreada com folhiço; 6=Moita de Tonina sp., $7=$ Tronco submerso.

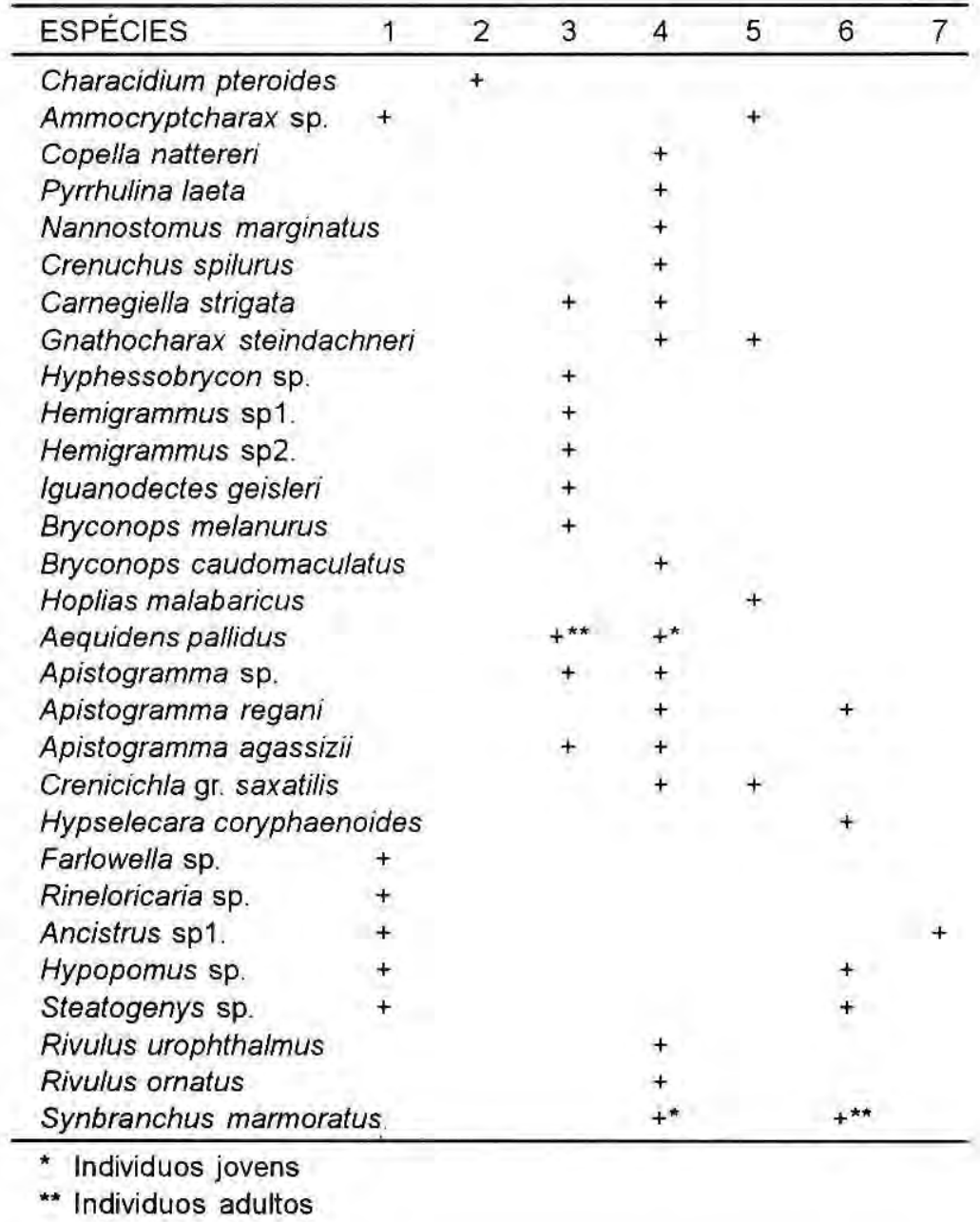

desenvolvidas, nas quais se apoía e permanece parada sobre o fundo.

Das onze espécies coletadas na água livre, sete tiveram ocorrência exclusiva neste biótopo: Hyphessobrycon sp., Hemigrammus spl, Hemigrammus $\mathrm{sp} 2,1$. geisleri, $B$. melanurus e $B$. caudomaculatus, qu s permaneceram nadando geralmente formando pequenos cardumes, e $A$. pallidus (adulto).
Nos alagados marginais, foram capturados principalmente indivíduos de pequeno porte. Todas as espécies que se alimentaram exclusivamente de itens alóctones ( $P$. laeta, $C$. strigata, $R$. ornatus e $R$. urophthalmus), bem como $C$. nattereri, $N$. marginatus, Crenuchus spilurus, e os exemplares jovens de $A$. pallidus e de Synbranchus marmoratus foram coletados somente nesta área; as 
outras espécies (C. strigata, Apistogramma sp., A. regani, $A$. agassizi, $C$. gr. saxatilis e Gnathocharax steindacneri) apareceram também em outros microhabitats.

Nas margens sombreadas foram coletadas $H$. malabaricus, $H$. coryphaenoides (só capturadas aqui), Crenicichla gr. saxatilis e Ammocryptocharax sp. (ambas com ocorrência também em outros biótopos).

Nos bancos de Tonina sp. foram capturados exemplares de $S$. marmoratus adulto, $A$. regani, Hypopomus sp. e Steatogenys sp.. Em tronco submerso, apenas Ancistrus sp. foi coletado.

Na Figura 2, é possivel observar a porcentagem relativa de espécies alimentando-se de itens alóctones e/ou autóctones nos diferentes microhabitats explorados. Nas moitas de Cyperaceae, a maioria das espécies presentes (75\%), alimentaram-se de itens autóctones e apenas Ammocryptocharax sp. $(25 \%)$ ingeriu alimentos de ambas origens. No fundo arenoso, a espécie C. pteroides foi a que predominantemente explorou esta área e consumiu preferencialmente itens autóctones (invertebrados bentônicos) $(100 \%)$. Em água livre, as espécies se alimentaram de itens autóctones e alóctones, perfazendo um total de $77,8 \%$ Elas apresentavam, de maneira geral, corpo fusiforme, o que thes garantiram grande mobilidade em toda coluna d'água. Das espécies presentes nos alagados marginais, $44,4 \%$ ingeriram somente itens alóctones, $22,2 \%$ alimentaram-se apenas de itens autóctones e 33,3\% ingeriram alimentos de ambas origens. Nas margens sombreadas, apenas $H$. malabaricus se alimentou exclusivamente de itens autóctones $(33.3 \%)$, o restante $(66,7 \%)$ consumiu concomitantemente alimentos de ambas origens. Todas as espécies presentes nos bancos de Tonina sp., se alimentaram $100 \%$ de itens autóctones, entretanto houve ocorrência de restos de insetos não identificados no conteúdo estomacal destas espécies. Em troncos submersos, Ancistrus sp., a única espécie coletada neste microhabitat, alimentou-se $100 \%$ de itens autóctones.

\section{DISCUSSÃO}

Grande diversidade de microhabitats foi observada na área de estudo. A maioria das espécies apresentou preferência por um dos biótopos observados e é possivel que o fator alimentação tenha sido a causa dessa preferência.

Em nosso trabalho, alguns peixes, apesar de não estarem ingerindo um único tipo de alimento, tiveram no seu conteúdo estomacal, itens oriundos de um determinado local como por exemplo as espécies $P$ laeta, $C$. strigata, $R$. urophthalmus e $R$ ornatus, que consumiram apenas itens acumulados nos alagados marginais. Fato semelhante foi também observado para C. pteroides que, mesmo apresentando espectro alimentar relativamente variado, os alimentos ingeridos eram quase todos invertebrados bentônicos. Os loricarídeos que foram coletados em 


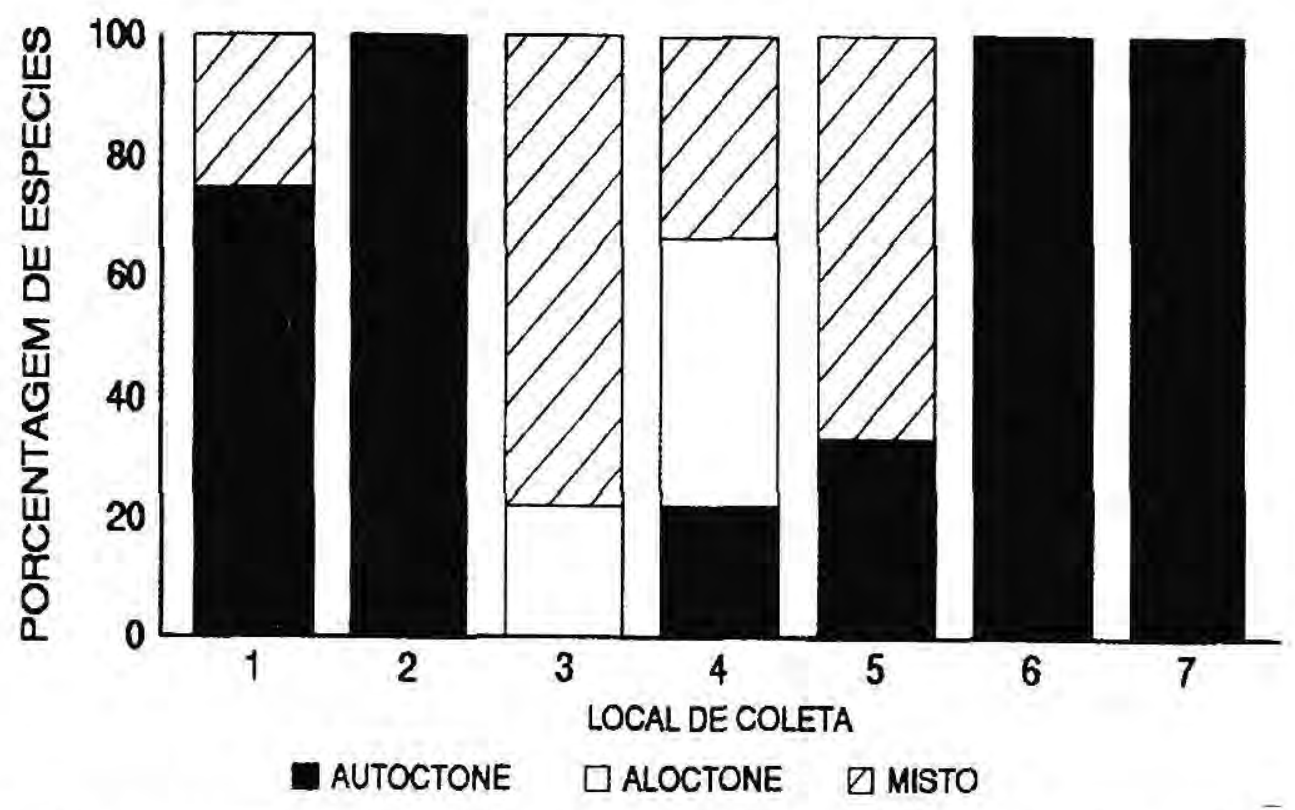

Figura 2. Percentagem do número de espécies, por origem do alimento consumido, por local de coleta. Os números relativos aos locais de coletas estão explicitos na tabela 3.

bancos de Cyperaceae, consumiram apenas algas filamentosas. Observamos, portanto, que nestes casos, não houve preferência alimentar por uma espécie de organismo em particular, mas por um grupo de organismo peculiar a um certo biótopo, no qual a
WALKER (1990), trabalhando em um igarapé de água preta na Amazônia Central, postularam que algumas espécies possuiam a distribuição altamente localizada e que também viviam e se alimentavam dentro de regiões restritas, em apenas poucos metros. LOWE-McCONNELL (1987) também observou que os peixes de zona tropical, tendiam a procurar seus 
alimentos dentro de microhabitats particulares. A existência de uma relação entre a alimento consumido e o microhabitat no qual a espécie vivia foi observada em nosso estudo. A dieta dos peixes, portanto, representa uma interação entre a preferência pelo alimento, a disponibilidade e a acessibilidade a esse alimento (ANGEMEIER \& KARR, 1984) e muitos ítens são característicos de habitats particulares (ZARET \& RAND, 1971).

As características morfológicas são consideradas especializações adaptativas das espécies (KEAST \& WEBB, 1966), havendo, portanto, uma interação entre o seu alimento e o seu local de vida. Neste estudo, apesar de não serem usadas medidas morfológicas no delineamento dos nichos das espécies, através da observação de simples estruturas morfológicas foi possivel notar que estas respondem tanto às necessidades tróficas quanto às de uso de um tipo de substrato. As espécies citadas acima, bem como outras que foram capturadas em água livre (os Characidae, por exemplo) apresentaram padrões morfológicos que condiziam com o tipo de alimento e o microhabitat onde foram capturadas. As espécies comuns na água livre apresentaram a dieta relativamente variada e se alimentaram tanto de ítens alóctones quanto autóctones. Este fato pode ser devido à grande mobilidade dessas espécies, o que permite que elas procurem alimento em toda coluna d'água. Esta relação foi também mencionada por outros autores (ZARET \& RAND, 1971; SHOENER, 1974;
GORMAN \& KARR, 1978; GATZ, 1979; PAINE et. al., 1982; WERNER, 1984; LOWE-McCONNELL, 1987).

As diferenças nas caracteristicas morfológicas ou no tamanho do corpo entre espécies tem sido utilizadas como indices de diferenças no uso de recursos espaciais e tróficos (KEAST \& WEBB, 1966; WERNER, 1984). SHOENER (1974) mencionou que os padrões morfológicos das espécies poderiam ser utilizados para avaliar as diferenças no uso de recurso entre elas. GATZ (1979) também postulou que a utilização de recursos estava relacionada com o fenótipo das espécies e que os peixes se especializavam em seus hábitos de acordo com suas adaptações morfológicas.

Outro fato observado neste estudo foi que algumas espécies apresentaram mudanças no padrão de utilização de microhabitat em relação ao seu estágio de desenvolvimento. KARR et. al. (1982) afirmaram que a seleção de microhabitat pelos peixes, pode variar com a idade, sexo, estágio reprodutivo, área geográfica $\mathrm{e}$ condições ambientais. As espécies $A$. pallidus e $S$. marmoratus, quando jovens $(3,5 \mathrm{~cm}$ e $7,5 \mathrm{~cm}$ de comprimento padrão, respectivamente) predominaram nos alagados marginais, onde permaneciam escondidas entre as gramineas, enquanto indivíduos maiores de $A$. pallidus $(>5,5 \mathrm{~cm}$ de comprimento padrão) foram geralmente coletados em água livre. Exemplares maiores que $15 \mathrm{~cm}$ de $S$. marmoratus passaram a colonizar bancos de Tonina sp. em áreas mais profundas. Essas 
mudanças, provavelmente não foram determinadas por alterações nas dietas dessas espécies, pois através da análise do conteúdo estomacal de vários individuos de tamanhos diferentes, não foram observadas mudanças. A base de alimento consumido foi praticamente a mesma para individuos grandes e pequenos de ambas espécies. A mudança de microhabitat provavelmente tenha sido um comportamento visando diminuir o risco de predação, já que nos alagados marginais, a pouca profundidade inviabilizava proteção para indivíduos maiores. Fato semelhante foi mencionado por POWER (1984) quando observou que exemplares grandes de Loricariidae evitavam águas rasas no intuito de fugir da predação, muito embora essas áreas fossem os locais onde havia grande quantidade de algas comumente ingeridas por eles. Os animais naturalmente apresentam o instinto de sobrevivência, ou seja, somente sobrevive aqueles que podem evitar a predação. Isto tem sido observado para peixes (WERNER et. al., 1983, ENDLER, 1987; COPP, 1990) bem como para outras taxa aquáticas (STRONG, 1973, BRONMARK et. al., 1984; $\mathrm{SIH}, 1988)$.

COPP (1990) estudando o comportamento de Rutilus rutilus (Cyprinidae) observou mudanças na utilização de microhabitat entre larvas e juvenis desta espécie, e postulou que essa mudança era um refúgio contra predação quando : s estruturas de proteção tornavam-se escassas ou não disponíveis. Ele também observou que as larvas, a partir do estágio $\mathrm{L}_{3}$ (ele classificou os estágios larvais de $\mathrm{L}_{1}$ a $\mathrm{L}_{6}$ ) apresentavam maior mobilidade e capacidade de escolha de microhabitat e isso coincidiu com a mudança na dieta, o que o levou a levantar a hipótese da alimentação como outro fator influenciando a troca de microhabitat. Em nosso estudo não observamos mudanças na dieta alimentar das duas espécies, o que nos leva a supor que, neste caso a diminuição de risco de predação foi a razão mais provável para a troca de microhabitat. WERNER et. al. (1983) também observaram mudanças ontogenéticas de nicho para muitas espécies de peixes e sugeriram que o risco de predação provavelmente atue fortemente sobre a "decisão" da espécie em onde permanecer e onde se alimentar.

Estudos que visem uma explicação dos possíveis fatores condicionantes da distribuição das espécies em microhabitats diferentes ainda são poucos. CONNELL (1975) citou três prováveis fatores: 1) alimentação, 2) intensidade de predação e 3) tolerância às condições físico-químicas. Para algumas espécies que nós observamos, é provável que a alimentação tenha sido o fator mais marcante, já que notamos uma interação entre o alimento consumido por certas espécies e seu constante local de captura. Para outras, a predação leva-nos a crer que seja a causa principal atuante na escolha do microhabitat, embora estudos mais detalhados nesta área sejam necessários. Em todos os casos observados, os parâmetros físico- 
químicos parecem não atuar como fatores limitantes na distribuição, o que significa dizer que as condições eram adequadas para toda a comunidade de peixes. SILVA (1992) demonstrou que as condições físico-químicas da água deste igarapé eram estáveis e típicas de sistemas aquáticos naturais.

De modo geral, as espécies de peixes tendem a passar grande parte de seu tempo de vida em um determinado microhabitat, isto porque a força evolutiva atua constantemente sobre os peixes, no intuito de criar especialistas espaciais, já que para os indivíduos, é conveniente assegurar a habilidade de encontrar e permanecer em um local dentro de um habitat (HENDERSON \& WALKER, 1990).

É provável que tal especialização não seja influenciada apenas por um único fator, e sim por um conjunto deles que leva as espécies a desenvolverem comportamentos que visem a utilização máxima de recursos e desta maneira, melhores condições de sobrevivência e perpetuação para a espécie.

\section{Bibliografia Citada}

ANGERMEIER, P. L \& KARR, J. R. (1984) Fish communities along environmental gradients in a system of tropical stream. In: ZARET, T. M. (ed.). Evolutionary Ecology of Neotropical Freshwater Fishes. Dr. Junk Publishers. The Hague, Netherland. 39-57.

BRONMARK, C.; MALMQVIST, B. \& OTTO, C. (1984) Antipredator adaptations in a neustonic insect Velia capria. Oecologia, 61: 189-191.

CHAUVEL, A. (1981) Contribuição para o estudo da evolução dos latossolos amarelos distróficos argilosos na borda do platô, na região de Manaus: mecanismo de gibbsitização. Acta Amazonica, 11(2): 227-245.

CHU, H. F. (1949) How to Know The Immature Insects. Wm. C. Brown Company Publishers. Dubuque Iowa, USA. $234 \mathrm{pp}$.

CODY, M. L. (1968) On the methods of resource division in grassland bird communities. Am. Nat. 102(924): 107-147.

CONNELL, J. H. (1975) Some mechanisms producing structure in natural communities: a model and evidence from field experiments. In: CODY, M. L. \& DLAMOND, J. M. (eds.). Ecology and evolution of communities. Belknap Press, Cambridge. 460-490.

COPP, G. H. (I990) Shifts in the microhabitat of larval and juvenile roach, Rutilus rutilus (L.), in a floodplain channel. $J$. Fish Biol., 36: 683-692.

COSTA, W. (1990a) Análise filogenética da familia Rivulidae (Cyprinodontiformes, Aplocheiloidei). Rev Bras. Biol. 50(1): 83-89.

COSTA, W. (1990b) Classificação e distribuição da familia Rivulidae (Cyprinodontiformes, Aplocheiloidei). Rev Bras. Biol. 50(1): 5-82.

ENDLER, J. A. (1987) Predation, ligth intensity and courtship behaviour in Poecilia reticulata (Pisces: Poeciliidae). Animal Behaviour, 35: 1376-1385.

GATZ, Jr. A. J. (1979) Community organization in fishes as indicated by morphological features. Ecology, 60(4):711-718.

GÉRY, J. (1977) Characoids of the World. T. F. H. Publications, New Jersey, 672pp

GOODALL, J. H. (1986) Biotope, estruture and patterning. In: KIKKAWA, J. \& ANDERSON D. J. (eds.) Community Ecology, Pattern and Process. Blackwell Scientific Publications. Australia. $30-40$

GORMAN, O. T. \& KARR, J. R. (1978) Habitat structure and stream fish communities. Ecology, 59: 507-515. 
HENDERSON, P. A \& WALKER, I (1990) Spatial organization population density of the fish community of the litter banks within a central Amazonian blackwater stream. J. Fish Biol. 37: 401-411.

KARR, J. R.; TOTH, L. A. \& GARMAN, G. D. (1982) Habitat preservation for midwest stream fishes: principles and guidelines. U. S. Environmental Protection Agency 600/3-83-006, Corvalis. $120 \mathrm{pp}$.

KEAST, A. \& WEBB, D. (1966) Mouth and body form relative to feeding ecology in the fish fauna of a small lake, Lake Openicon, Ontario. J. Fish. Res. Bd. Canada, 23(12): 1845-1874.

KULLANDER, S. O. (1986) Cichlid fishes of the Amazon river drainage of Peru. Museum of Natural History, Stockolm. $431 \mathrm{pp}$

LOWE-McCONNELL, R. H. (1975) Fish communities in tropical freshwater their distribution ecology and evolution. London Logman 377pp

LOWE-McCONNELL, R. H. (1987) Ecological studies in tropical fish communities. Cambrigde University Press. $382 \mathrm{pp}$.

MACAN, T. T. (1959) A Guide to Freshwater Invertebrates Animals. Longman. $118 \mathrm{pp}$.

PAINE, M. D; DODSON, J. J. \& POWER, G. (1982) Habitat and food resource partitioning among four species of darters (Percidade: Etheostoma) in a southern Ontario stream. Can. J. Zool. 60: 1335-1341.

POWER, M. E. (1984) Grazing responses of tropical freshwater fishes to different scales of variation in their food. In: ZARET, T. (ed,) Evolutionary Ecology of Neotropical Freshwater Fishes. Dr. W. Junk Publishers, The Hague . 25-37.

RADAMBRASIL (1978) Mapas do Projeto Radam. Departamento Nacional de Produtos Minerais.

RIBEIRO,M. N. G \& ADIS, J. (1984) Local rainfall variability - a potencial bias for bioecological studies in the Central
Amazon. Acta Amazonica, 14(1/2): 159174

RINNE, J. N. (1992) Physical 'tabitat utilization of fish in a Sonoran Desert stream,Arizona, southern United States. Ecology of Fresh, ater Fish 1; 35-41.

SANTOS, G. M (1990) Inventário taxonômico e ecologia dos peixes dos rios Jamari, Machado, Guaporé, Mamoré e Pacaás Novos (Rondônia). Tese de doutorado. INPA/FUA 228pp.

SAUL, W. G. (1975) A ecologycal study of fishes at a site in Upper Amazonian Ecuador. Proc. Acad. Nat. Sci. Philadelphia, 127: 93-134.

SHOENER, T. W. (1974) Resource partitioning in ecological communities. Science, 185: 27-39.

SIH, A. (1988) The effects of predators on habitat use, activity and mating behaviour of a semi-aquatic bug. Animal Behaviour, 36: 1846-1848.

SILVA, C. P. D (1992) Influência das modificações ambientais sobre a comunidade de peixes de um igarapé na cidade de Manaus (Amazonas). Dissertação de Mestrado. INPA/FUA, Manaus. $112 \mathrm{pp}$.

SOARES, M. G. M. (1979) Aspectos ecológicos (alimentação e reprodução) dos peixes do igarapé do Porto, Aripuanã, MT. Acta Amazonica, 9(2): 325-352.

STRONG, D. R. (1973) Amphypod amplexus, the significance of ecotypic variation. Ecology, 54: 1383-1388.

VIANNA, J. P. (1989) Estrutura de comunidade dos peixes do Ribeirão Sant'Ana (Brasilia-DF) ao longo de gradientes ambientais. Dissertação de mestrado. $115 \mathrm{pp}$.

WERNER, E. E. (1984) The mechanisms of species interactions and community organization in fish. In: STRONG, JR. D. R; SIMBERLOFF, D.; ABELE, L. G. \& THISTLE, A. B. (eds.). Ecological Communities: conceptual issue and evidence. Princeton University Press, New Jersey, U.S.A. 360-382. 
WERNER, E. E., GLLIAM, J. F; HALL, D. I. \& MITTELBACH, G. G. (1983) An experimental test of the effects of predation risk on habitat use in fish. Ecology, 64(6): $1540-1548$.
ZARET, T. M. \& RAND, A. S. (1971) Competition in tropical stream fishes; support for the competitive exclusion principle. Ecology, 52(2): 336-342 